\title{
OPEN High order Fano resonances and giant magnetic fields in dielectric microspheres
}

\author{
Zengbo Wang ${ }^{1 *}$, Boris Luk'yanchuk ${ }^{2,3^{*}}$, LiyangYue ${ }^{1}$, Bing Yan $^{1}$, James Monks ${ }^{1}$, \\ Rakesh Dhama ${ }^{1}$, Oleg V. Minin ${ }^{4,5}$, IgorV. Minin ${ }^{4,5}$, Sumei Huang ${ }^{6}$ \& Andrey A. Fedyanin ${ }^{3}$
}

We show that weakly dissipating dielectric spheres made of materials such as glass, quartz, etc. can support high order Fano resonances associated with internal Mie modes. These resonances, happening for specific values of the size parameter, yield field-intensity enhancement factors on the order of $10^{4}-10^{7}$, which can be directly obtained from analytical calculations. Associated to these "superresonances", we analyze the emergence of magnetic nanojets with giant magnetic fields, which might be attractive for many photonic applications.

Fano resonances, resulting from the interference of broad and narrow excitation modes, have emerged as an interesting topic of research with promising applications in physical, chemical, and biological sciences ${ }^{1,2}$. The quality factor (sharpness), as well as the amplitude of Fano resonances increase for higher order modes. As an example, interference of a broad dipole (Rayleigh-like) mode and a narrow octupole mode provides a sharper resonance than that stemming from the interference between the dipole and quadrupole modes in plasmonic particles (see e.g. Fig. 3 in ref. ${ }^{1}$ ). Therefore, excitation of these, higher-order Fano resonances ${ }^{3-6}$ might be beneficial to enhance the sensitivity of resonant nanostructures. An example of such high order Fano resonances (quadrupole, octupolar, hexadecapolar, and triakontadipolar) are those generated in optimized disk-ring silver plasmonic nanostructure $^{6}$. Further progress towards higher order resonances in plasmonic nanostructures, however, has been limited by the large dissipation associated with metals, particularly in the visible range. On the contrary, dielectric materials might be the perfect platform to observe such effects, as the dissipation effects can be very small. In the following, we show that this is the case, and that realization of high order Fano resonances is possible in simple systems such as spherical particles, including both high index (e.g. $\mathrm{n}=4$ ) and moderate (e.g. $\mathrm{n}=1.5$ ) index materials.

To study the emergence of high-order resonances in spherical particles, we make use of the well-known solution of the scattering problem from a homogeneous sphere, i.e., the Mie theory. A brief summary of the main expressions allowing to compute the scattering $\left(Q_{\ell}\right)$ and internal $\left(F_{\ell}\right)$ fields associated to the different electric and magnetic multipolar modes supported by the particle $\left(\ell\right.$ being the order) are given in the Methods section ${ }^{7-9}$. In Fig. 1 we present the characteristic positions of different resonances and maximal values of the corresponding scattering and internal field amplitudes $Q_{\ell}$ and $F_{\ell}$ for a spherical particle with refractive index $n=4$. For modes above octupolar order $(\ell \geq 3)$ resonances are very sharp, and we show them in the right panel of Fig. 1 with higher resolution of the size parameter $q$. The observed fast increase in the amplitude of the internal resonances is accompanied by a huge growth of the electric and magnetic fields, as shown in Fig. 2. In particular, Fig. 2a,b display the intensity of electric and magnetic fields inside the particle at the electric and magnetic octupolar resonances, respectively. As can be seen, the enhancement of the magnetic field intensity can reach values that are up to two orders of magnitude larger than those of the electric field. For completeness, Fig. $2 \mathrm{c}$ shows the comparison between the maximal values of the magnetic field intensity inside the particle and at its surface, for the first four resonances supported. In the following, we extend this intensity enhancement effect to higher order resonances using moderate refractive index materials that are known to have very low dissipative losses, such as glass.

\footnotetext{
${ }^{1}$ School of Electronic Engineering, Bangor University, Dean Street, Bangor, Gwynedd, LL57 1UT, UK. ${ }^{2}$ Division of Physics and Applied Physics, School of Physical and Mathematical Sciences, Nanyang Technological University, Singapore, 637371, Singapore. ${ }^{3}$ Faculty of Physics, Lomonosov Moscow State University, Moscow, 119991, Russia. ${ }^{4}$ National Research Tomsk State University, Lenin Ave., 36, Tomsk, 634050, Russia. ${ }^{5}$ National Research Tomsk Polytechnic University, Lenin Ave., 30, Tomsk, 634050, Russia. ${ }^{6}$ Engineering Research center for Nanophotonics \& Advanced Instrument, Ministry of Education, School of Physics and material Science, East China Normal University, Shanghai, 200062, PR China. *email: z.wang@bangor.ac.uk; lukiyanchuk@nanolab.phys.msu.ru
} 


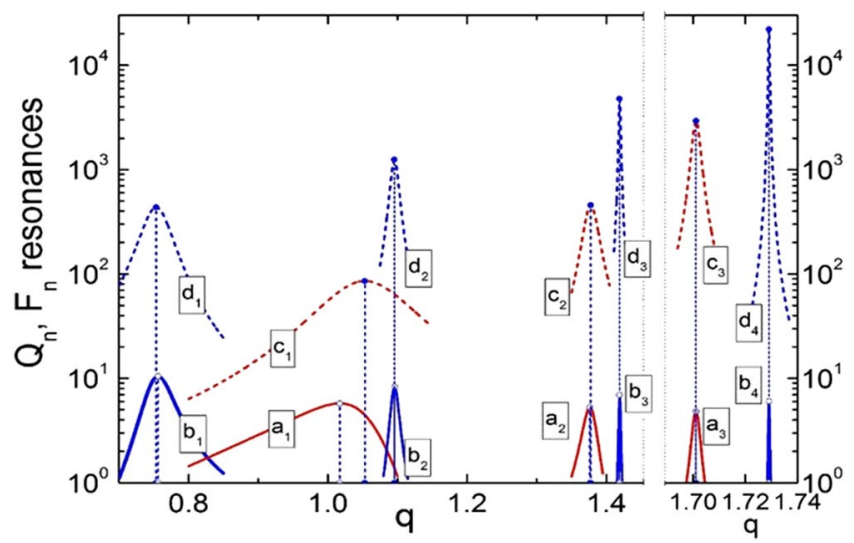

Figure 1. Partial scattering efficiencies $Q_{\ell}$ and $F_{\ell}$, related to scattering amplitudes, $a_{\ell}$ and $b_{\ell}$, and internal field amplitudes, $c_{\ell}$ and $d_{\ell}$, for a spherical particle with refractive index $n=4$ as a function of its size parameter $q$. Solid lines represent the partial scattering efficiencies $Q_{\ell}$, and dotted lines the internal partial efficiencies $F_{\ell}$. Electric amplitudes are shown in red color and magnetic amplitudes in blue. Note the change in the $\mathrm{x}$-axis scale, introduced to present the sharp resonances found for $\ell \geq 3$.
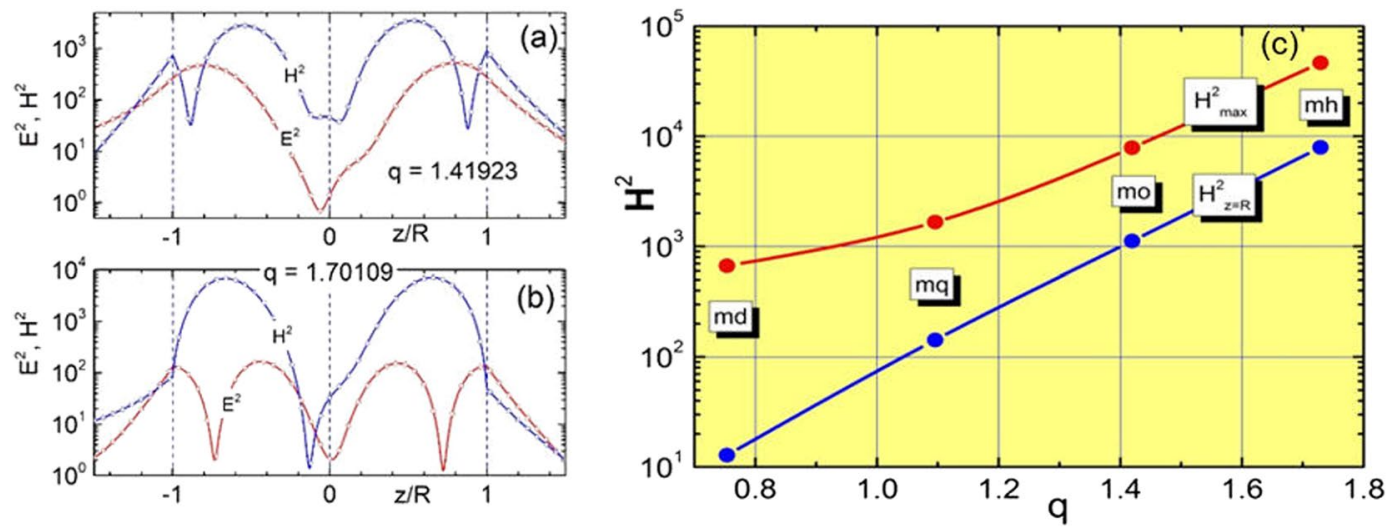

Figure 2. Electric and magnetic field distributions for a particle with refractive index $\mathrm{n}=4$ and size parameter (a) $q=1.41923$ (magnetic octupole resonance) and (b) $q=1.70109$ (electric octupole resonance). (c) Maximal values of $\mathrm{H}^{2}$ (normalized to the incidence ones) inside the particle (red dots) for the first four internal magnetic resonances. The intensity of the magnetic field at the particle surface, in the point opposite to the wave incidence, are also shown (blue dots). Smooth solid lines are shown just for better visibility. Particle refractive index $n=4$.

\section{High Order Fano Resonances}

The microscopic origin of the Fano resonance arises from the constructive and destructive interference of a narrow discrete resonance with a broad spectral line or continuum ${ }^{1,2}$. In our case, we are interested in studying Fano resonances arising from the interference between high order resonances and the broad spectrum provided by all the other modes. In Fig. 3, we illustrate this effect for the particle with refractive index $n=1.5$ (non-absorbing, close the typical values of glasses) and size parameter $q=26.9419$. These parameters correspond to a resonant magnetic mode excited inside the particle with partial wave order $\ell=35$. At the same time the total number of modes with a significant contribution for this size parameter in the Mie theory is $\ell_{\max }=41$. In Fig. 3a, one can see the electric field intensity distribution in the $\{x, z\}$ plane, where all modes with $\ell \leq \ell_{\max }$ are taken into account in calculations. In Fig. $3 \mathrm{~b}$ we present the same picture where all $\ell$ terms are taken into account except the single resonant term with $\ell=35$. From the comparison one can immediately see that the single term $\ell=35$ produces around a 200 times increase of the intensity.

Due to the interference of broad and narrow spectral lines, we obtain characteristic Fano line-shapes in the far field scattering as well as for the electric and magnetic intensity spectrums, as shown in Fig. 4 for some size parameter range near $q \approx 20$. The emergence of the Fano shape in the scattering efficiency was analyzed in Ref. ${ }^{10}$. These extra narrow resonances produce about $20 \%$ variation of signal in the scattering efficiency, but one-two orders of magnitude in the electric and magnetic field intensities. In Fig. $4 \mathrm{~b}$, we show the intensities spectra on the surface of the particle, i.e. at $\{x=y=0, z=R\}$. The wave is taken as propagating in the positive z-axis. Note that the intensities inside the particle can be up to one order of magnitudes higher, as can be seen, e.g., in Fig. 3a. We coin these high order Fano resonances, for which field-intensity enhancement factors can reach values on the 

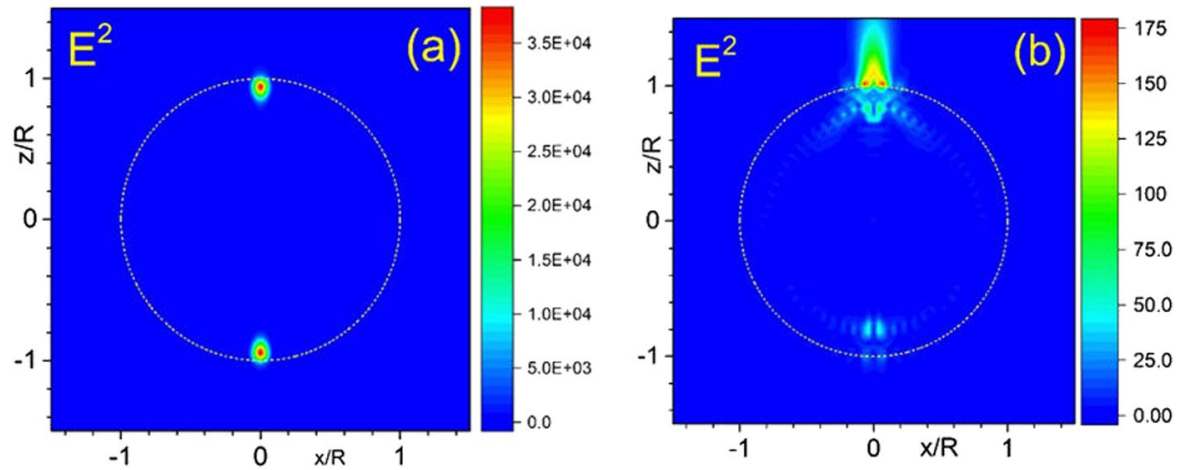

Figure 3. Distribution of intensity $E^{2}$ in the $\{x, z\}$ plane, calculated by Mie theory, where all terms $\ell \leq \ell_{\max }=41$ are taken into account in calculations (a) and the same distribution without the single $\ell=35$ resonant term (b).
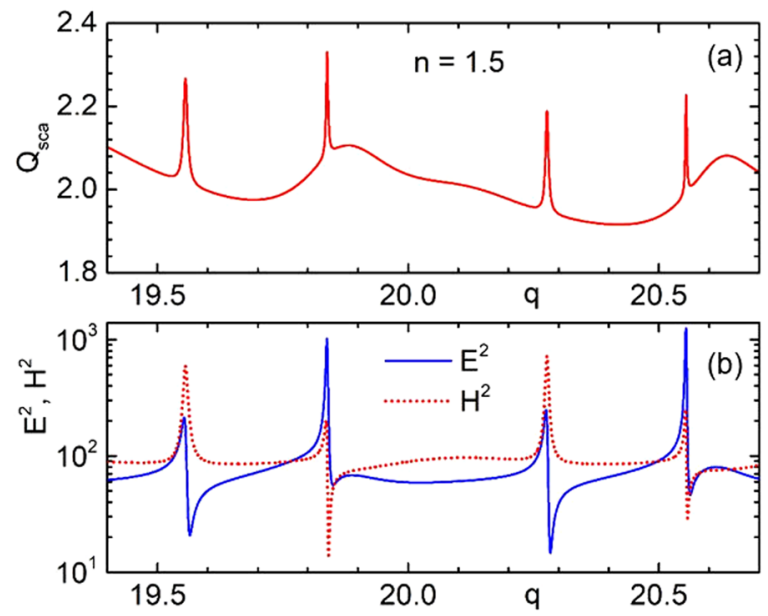

Figure 4. Scattering efficiency $Q_{s c a}(q)$ for particle with refractive index $n=1.5$ near size parameter $q \approx 20$ (a). Electric and magnetic intensities on the surface for the same particle (b).

order of $10^{4}-10^{7}$, as "super resonances". Note that the typical range of size parameters necessary to obtain such resonances obviously depend on the refractive index. For the moderate values of highly transparent materials like glass, the range of interest lies within $q \approx 10 \div 30$. In order to obtain these effects in the visible range of the spectrum, one should thus consider particle sizes from a few to few-tens of micrometers.

Another important feature of high order Fano resonances is the high degree of field localization (beyond the diffraction limit) inside the particle and on the particle surface, as seen for example in Fig. 5. This is related to the formation of regions with high values of local wave vectors, an effect that has been discussed, e.g., in the frame of the theory of superoscillations ${ }^{11-21}$. According to this theory, the local wave vector can be understood as the local gradient of the phase $\mathbf{k}_{\text {local }}=\nabla \Phi=\nabla \mathbf{E} / E$. High values of $k_{\text {local }}$ can be created, e.g., by metamaterial lenses, by phase and amplitude masks and also in free space optics including vortices and knots. An optical vortex presents a singularity (zero intensity point) with circulating field phase around, given by a topological number $n$ (total change of phase around singularity is $2 \pi n$ ). Taking a path with radius $r$ around the singular point one can see that the change of $2 \pi n$ in phase occurs along the length $2 \pi r$, i.e. the corresponding gradient of phase has an order $n / r$, which means that local wave vector tends to infinity at $r \rightarrow 0$. This property also follows from the energy-time form of the Heisenberg uncertainty principle $\Delta E \cdot \Delta t \geq \hbar / 2$. Using $E=N \hbar \omega$ and $t=\Phi / \omega$ this uncertainty can be reformulated in terms of number of photons and their phase $\Delta N \Delta \Phi \geq 1 / 2$. Differentiating this formula is easy to find that $k_{\text {local }} \propto \nabla N / N^{2}$, i.e. high local wave numbers can be reached in the vicinity of the singularity.

Optical nanovortices can be created around plasmonic and dielectric nanoparticles ${ }^{22-29}$. In dielectric particles optical vortices arise when size parameter exceeds some value, which depends on its refractive index ${ }^{30,31}$. These vortices can be easily seen in the plot of Poynting's vector, as seen, for example, in Fig. 6. It corresponds to a distribution of displacement currents in $\{x, z\}$ plane. Circular currents create magnetic fields according to the Biot-Savart law, which states that the magnetic field at center of a current loop is given by $B=\mu I / 2 R$. For a current $I=1 \mathrm{~A}$ and a loop with radius $R=10 \mathrm{~nm}$ one can create magnetic induction $B=63.8$ Tesla (T)

In Fig. 6 one can see vortices with size $\lambda / 10 \pi$. Loops of displacement current are of the order of $20 \mathrm{~nm}$ in visible range. It shows the ability to create high magnetic fields above $10 \mathrm{~T}$ even with small silicon particles. High 


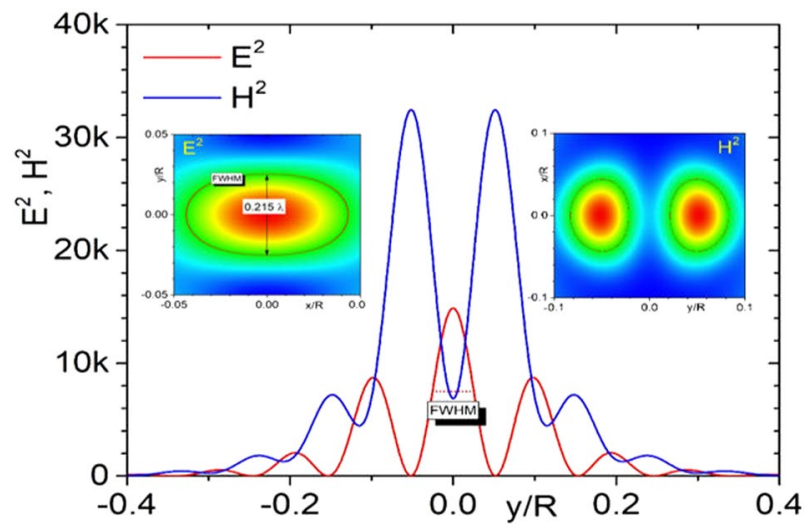

Figure 5. Distribution of the electric $E^{2}$ and magnetic $H^{2}$ fields along the $y$-axis at the output plane at $z=R$ for particle with refractive index $n=1.5$ and size parameter $q=26.9419$. It corresponds fields variations along the $y$-coordinate along the upper bright point in Fig. 3a. 2D field distributions within the $\{x, y\}$ planes are shown in the insets. The solid lines in the $2 \mathrm{D}$ insets indicate the position of the full width at half maximum (FWHM). One can see high light localization along the y-direction: for electric field the FWHM is $0.215 \lambda$, less than the diffraction limit given by $\lambda / 2 n=0.333 \lambda$.

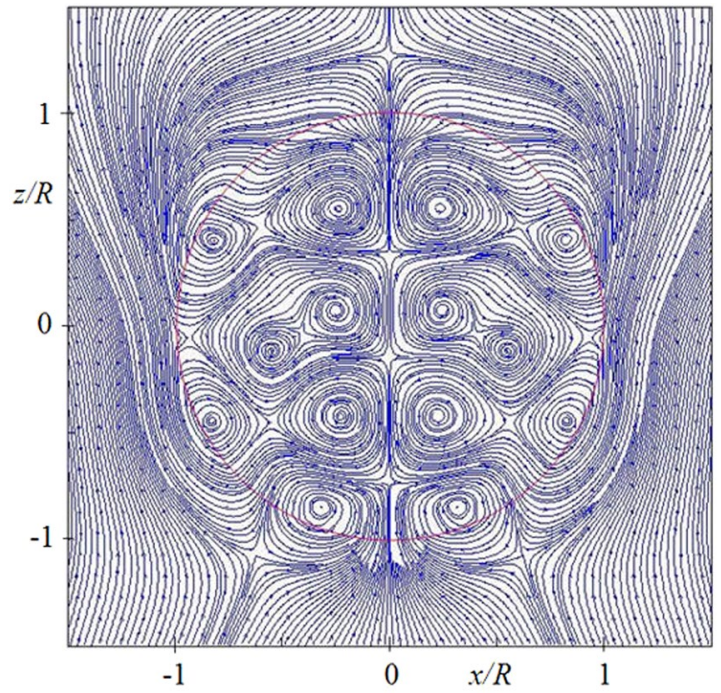

Figure 6. Poynting vector distribution for the particle with refractive index $n=4$ and $q=2$.

enhancement of localized electric and magnetic fields with small silicon nanodimer was experimentally demonstrated in Ref. ${ }^{32}$. Calculation results show the enhancement of magnetic field was twice larger than the electric field enhancement ${ }^{32}$. Note that magnetic fields of light are quite small. For example, $1 \mu \mathrm{J}, 100 \mathrm{fs}$ laser pulse focused to a $200 \mu \mathrm{m}^{2}$ area produces $5 \times 10^{16} \mathrm{~W} / \mathrm{cm}^{2}$ or electric fields of the order of $10^{7} \mathrm{~V} / \mathrm{cm}$, as reported in Ref. ${ }^{33}$. This yields magnetic induction in vacuum of just about $3 \mathrm{~T}$. However, inside a glass particle, as shown above, magnetic field can be enhanced above $3 \cdot 10^{4}$ times (see in Fig. 5), which may produce magnetic induction values of the order of $10^{5} \mathrm{~T}$ for the same exciting laser (close to interatomic magnetic fields). With this field one can expect nonlinear dependence $\mu=\mu(H)$, i.e. magnetic nonlinear optics, where variations of the refractive index $n=\sqrt{\varepsilon \mu}$ is caused by purely magnetic effects. Such magnetic nonlinear optics can be realized if two conditions are fulfilled: 1) the dissipation is quite small and 2) the magnetic nonlinear response significantly exceeds the electric nonlinear response due to non-linearity $\varepsilon=\varepsilon(E)$.

It is not easy to fulfill these two conditions. First, super resonances are quite sensitive to dissipation. For example, in Fig. 7 we show the evolution of a magnetic super resonance for a particle with refractive index $n=4$ and size parameter $q=4.4241$ as a function of the imaginary part of the refractive index. The fields in the case of a purely non-dissipative material at this super resonance reach values around $10^{6}$ (for electric intensity $E^{2}$ ) and $10^{7}$ (for magnetic intensity $H^{2}$ ). However, with as low dissipation as $\operatorname{Im} n \leq 10^{-3}$ these resonances are strongly suppressed. Probably, the necessary level of dissipation to see super resonances in the optical range can be reached for materials with refractive index less than two (e.g. glass). While it is questionable whether it is possible to realize 

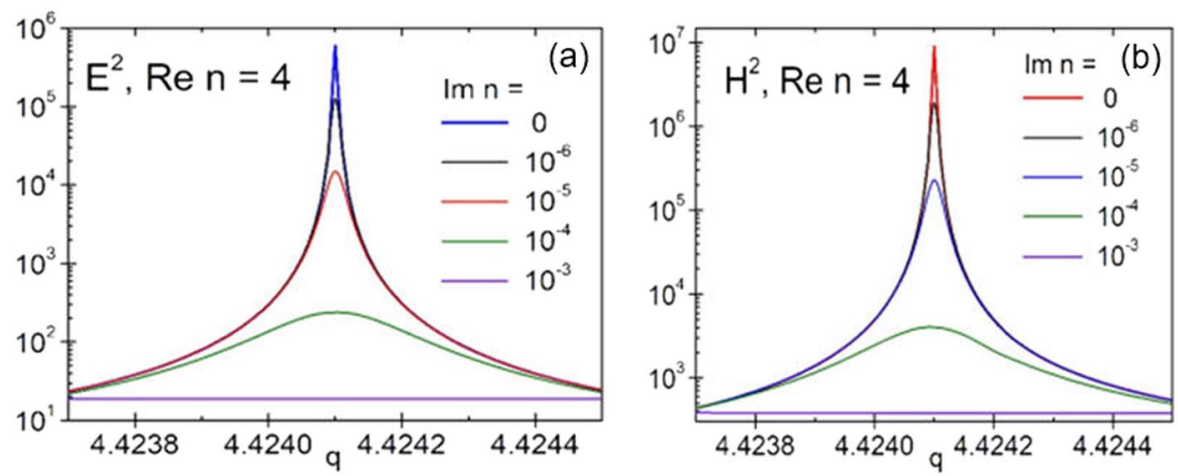

Figure 7. Effect of dissipation for the case of a magnetic super resonance excited in a particle with refractive index $n=4$ and size parameter $q=4.4241$. Dissipation of the level of $\operatorname{Im} n \approx 10^{-5}$ yields two order of magnitude suppression in the maximal field values, while at $\operatorname{Im} n \approx 10^{-3}$ these resonances practically disappear.
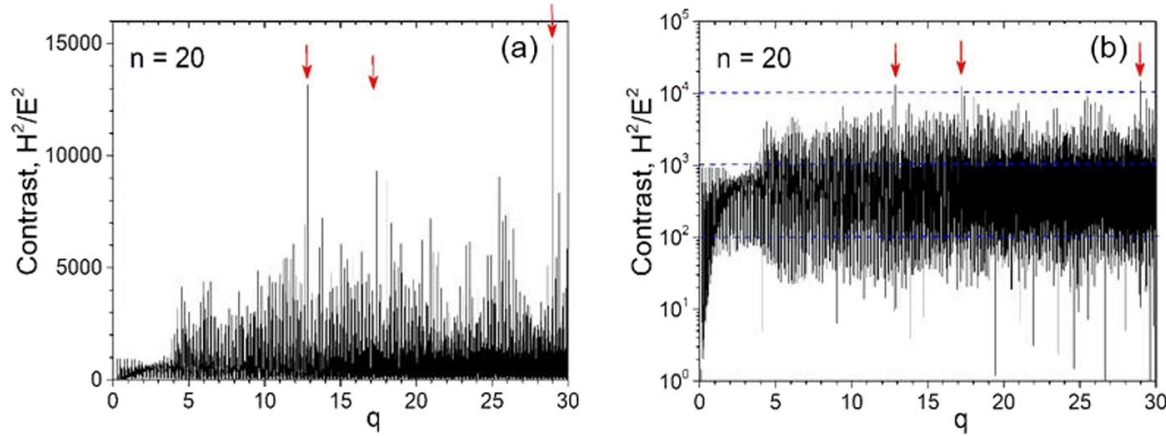

Figure 8. Contrast between magnetic and electric field enhancements for a particle with refractive index $n=20$ as a function of the size parameter presented in decimal (a) and logarithmic (b) scales.

such high order resonances using high-index material (e.g. Si) in the optical range, it maybe be still possible to do so in the IR range, where the level of dissipation can be very low.

The second condition means that we need a high contrast of magnetic to electric field enhancement, i.e. $H^{2} / E^{2} \geq 10^{4}$. Calculations show that this contrast is small (typically less than 10) for a particle with refractive index $n \leq 2$. Also, it decreases with the increase of the size parameter. However, with high refractive index it is possible to provide high contrast at some particular values of $q$, as shown in Fig. 8. There are three values of $q$ at which this can be achieved. Roughly speaking, for $H^{2} / E^{2}>10^{4}$ magnetic optical effects are dominant, for $10^{3} \leq H^{2} / E^{2} \leq 10^{4}$ both, electric and magnetic contributions in nonlinear effects are comparable, and for $H^{2} / E^{2} \leq 10^{3}$ conventional nonlinear optics related to nonlinearity $\varepsilon=\varepsilon(E)$ is dominant. Big values of $n$ for some ceramics ${ }^{34}$, e.g. $\varepsilon=180$ for $\mathrm{Ti}_{2} \mathrm{Nb}_{10} \mathrm{O}_{29}$ can be seen in the microwave region. However it is not clear how small dissipation can be reached. In any case, with super resonances in dielectrics, it might be possible to realize magnetization induced optical nonlinearity. Until now, nonlinear effects in magnetization-induced optical nonlinearity were observed mainly in thin ferromagnetic films $\mathrm{s}^{35,36}$.

Beyond their possible application in novel non-linear phenomena, the ability to create highly localized fields with enhancement values on the level of $10^{5}$, both, inside the particle and outside in the near field region, open new venues in many modern applications, such as in photonic magnetic nanojet generation ${ }^{31}$, white light superlens nanoscopy ${ }^{30}$ and surface-enhanced Raman spectroscopy (SERS $)^{37}$. Also, it might serve as a means for optimization of whispering gallery modes, widely used for telecommunication applications in, e.g., wavelength division multiplexing (WDM) schemes ${ }^{38,39}$.

\section{Conclusions}

In conclusion, we reveal novel, super-resonance modes supported by dielectric spheres. These resonances, present for all multipolar orders, exhibit Fano line shapes and have extraordinarily high associated electric and magnetic field enhancements, which increase linearly with the multipolar order. The phenomenon can be observed at visible frequencies using simple glass microspheres, which may allow enhancing the magnetic field of light (which is typically small) by a few orders of magnitude. We believe these super-resonances are an attractive platform for some promising applications, like e.g. enhanced absorption effect, ablation caused by magnetic pressure, or those mentioned above. 


\section{Methods}

The Mie theory express the scattered fields in the form of superposition of partial waves in terms of spherical harmonics ${ }^{7-9}$. According the Mie theory the total scattering efficiency is presented by sum of partial scattering efficiencies:

$$
Q_{s c a}=\sum_{\ell=1}^{\infty}\left(Q_{\ell}^{(e)}+Q_{\ell}^{(m)}\right), \quad Q_{\ell}^{(e)}=\frac{2(2 \ell+1)}{q^{2}}\left|a_{\ell}\right|^{2}, \quad Q_{\ell}^{(m)}=\frac{2(2 \ell+1)}{q^{2}}\left|b_{\ell}\right|^{2} .
$$

where each partial efficiency corresponds to the radiation of the $\ell$-th order multipole. Terms $Q_{\ell}^{(e)}$ and $Q_{\ell}^{(m)}$ describe the radiation related to the electric and magnetic polarizabilities, respectively. In the following, we will discuss transparent dielectrics with $\operatorname{Im} \varepsilon=0$, so $Q_{e x t}=Q_{s c a}$. The electric, $a_{\ell}$, and magnetic, $b_{\ell}$, scattering amplitudes for nonmagnetic materials with relative magnetic susceptibility $\mu=1$, and dielectric permittivity $\varepsilon=n^{2}(n$ being the refractive index of the particle material) are given by:

$$
a_{\ell}=\frac{\mathfrak{R}_{\ell}^{(a)}}{\mathfrak{R}_{\ell}^{(a)}+i \mathfrak{I}_{\ell}^{(a)}}, \quad b_{\ell}=\frac{\mathfrak{R}_{\ell}^{(b)}}{\mathfrak{R}_{\ell}^{(b)}+i \mathfrak{I}_{\ell}^{(b)}},
$$

where $\mathfrak{R}_{\ell}$ and $\mathfrak{I}_{\ell}$ functions are defined as follows:

$$
\begin{array}{ll}
\mathfrak{R}_{\ell}^{(a)}=n \psi_{\ell}^{\prime}(q) \psi_{\ell}(n q)-\psi_{\ell}(q) \psi_{\ell}^{\prime}(n q), & \mathfrak{I}_{\ell}^{(a)}=n \chi_{\ell}^{\prime}(q) \psi_{\ell}(n q)-\chi_{\ell}(q) \psi_{\ell}^{\prime}(n q), \\
\mathfrak{R}_{\ell}^{(b)}=n \psi_{\ell}^{\prime}(n q) \psi_{\ell}(q)-\psi_{\ell}(n q) \psi_{\ell}^{\prime}(q), & \mathfrak{I}_{\ell}^{(b)}=n \chi_{\ell}(q) \psi_{\ell}^{\prime}(n q)-\psi_{\ell}(n q) \chi_{\ell}^{\prime}(q) .
\end{array}
$$

Here, $\psi_{\ell}(z)=\sqrt{\frac{\pi z}{2}} J_{\ell+\frac{1}{2}}(z), \chi_{\ell}(z)=\sqrt{\frac{\pi z}{2}} N_{\ell+\frac{1}{2}}(z)$, where $J_{\ell+\frac{1}{2}}(z)$ and $N_{\ell+\frac{1}{2}}(z)$ are the Bessel and Neumann functions. The radius of the particle $k$ enters in this theory through the dimensionless size parameter $q=\omega R / c=2 \pi R / \lambda$, where $\omega$ is the angular frequency, $c$ the speed of light, and $\lambda$ the radiation wavelength in vacuum. The prime in formulas (3), (4) indicates differentiation with respect to the argument of the function, i.e. $\psi_{\ell}^{\prime}(z) \equiv d \psi_{\ell}(z) / d z$, etc. Electric and magnetic fields inside the particle are expressed through the internal scattering amplitudes given by

$$
c_{\ell}=\frac{\text { in }}{\mathfrak{R}_{\ell}^{(a)}+i \mathfrak{I}_{\ell}^{(a)}}, \quad d_{\ell}=\frac{\text { in }}{\mathfrak{R}_{\ell}^{(b)}+i \mathfrak{I}_{\ell}^{(b)}} .
$$

Although the denominators of these amplitudes are the same as in amplitudes $a_{\ell}$ and $b_{\ell}$ in (2), which means that position of these resonances are close, the numerators of (5) never tends to zero. As a result the values of amplitudes $\left|c_{\ell}\right|^{2}$ and $\left|d_{\ell}\right|^{2}$ are not restricted by unity as amplitudes $\left|a_{\ell}\right|^{2}$ and $\left|b_{\ell}\right|^{2}$ in (2), but increase with values of size parameter and refractive index. To compare both type of resonances it is convenient to introduce partial internal scattering efficiencies, like in Eq. (1):

$$
F_{\ell}^{(e)}=\frac{2(2 \ell+1)}{q^{2}}\left|c_{\ell}\right|^{2}, \quad F_{\ell}^{(m)}=\frac{2(2 \ell+1)}{q^{2}}\left|d_{\ell}\right|^{2} .
$$

Super-resonance modes are extremely sensitive to the size parameter $q$. Our tests reveal that a $q$ sampling accuracy of $10^{-4}$ is required to ensure all modes were identified. This in turn leads to a supercomputing problem and parallelization of Mie code is required. We used a specially designed 10-parallel-thread Mie code for the computation, which takes about 4 hours to complete the calculation as in Fig. 8(a).

Received: 23 August 2019; Accepted: 10 December 2019;

Published online: 30 December 2019

\section{References}

1. Luk'yanchuk, B. et al. The Fano resonance in plasmonic nanostructures and metamaterials. Nature Materials 9, 707-715 (2010).

2. Miroshnichenko, A. E., Flach, S. \& Kivshar, Y. S. Fano Resonances in Nanoscale Structures. Rev. Mod. Phys. 82, 2257-2298 (2010).

3. Hao, F., Nordlander, P., Sonnefraud, Y., Van Dorpe, P. \& Maier, S. A. Tunability of subradiant dipolar and Fano type plasmon resonances in metallic ring/disk cavities: implications for nanoscale optical sensing. ACS Nano 3, 643-652 (2009).

4. Sonnefraud, Y. et al. Experimental realization of subradiant, superradiant, and Fano resonances in ring/disk plasmonic nanocavities. ACS Nano 4, 1664-1670 (2010).

5. Dregely, D., Hentschel, M. \& Giessen, H. Excitation and tuning of higher-order Fano resonances in plasmonic oligomer clusters. ACS Nano 5, 8202-8211 (2011).

6. Fu, Y. H., Zhang, J. B., Yu, Y. F. \& Luk'yanchuk, B. Generating and manipulating higher order Fano resonances in dual-disk ring plasmonic nanostructures. ACS Nano 6, 5130-5137 (2012).

7. Van de Hulst, H. C. Light Scattering by Small Particles (Dover, New York, 2005).

8. Born, M. \& Wolf, E. Principles of Optics, 7th ed. (Cambridge University Press, Cambridge, 1999).

9. Bohren, C. F. \& Huffman, D. R. Absorption and Scattering of Light by Small Particles (Wiley, New York, 1998).

10. Tribelsky, M. I. \& Miroshnichenko, A. E. Giant in-particle field concentration and fano resonances at light scattering by highrefractive-index particles. Phys. Rev. A 93, 053837 (2016).

11. Berry, M. V. Five momenta. Eur. J. Phys. 44, 1337-1348 (2003).

12. Zheludev, N. I. What diffraction limit? Nature Materials 7, 420-422 (2008).

13. Aharonov, Y., Colombo, F., Sabadini, I. R., Struppa, D. C. \& Tollaksen, J. Some mathematical properties of superoscillations. J. Phys. A Math. Theor. 44, 365304 (2011).

14. Rogers, E. T. F. et al. A super-oscillatory lens optical microscope for subwavelength imaging. Nature Materials 11, 432 (2012). 
15. Rogers, E. T. F. \& Zheludev, N. I. Optical super-oscillations: sub-wavelength light focusing and super-resolution imaging. J. Opt. 15, 094008 (2013).

16. Berry, M. V. \& Moiseyev, N. Superoscillations and supershifts in phase space: Wigner and Husimi function interpretations. J. Phys. A Math. Theor. 47, 315203 (2014).

17. Huang, K. et al. Optimization-free superoscillatory lens using phase and amplitude masks. Laser \& Photonics Reviews 8, 152-157 (2014).

18. Berry, M. V. Superoscillations and leaky spectra. J. Phys. A Math. Theor. 52, 015202 (2018)

19. Berry, M. et al. Roadmap on superoscillations. J. Opt. 21, 053002 (2019).

20. Yuan, G., Rogers, E. T. F. \& Zheludev, N. I. "Plasmonics" in free space: observation of giant wavevectors, vortices, and energy backflow in superoscillatory optical fields. Light: Science \& Applications 8, 2 (2019).

21. Yuan, G. H. \& Zheludev, N. I. Detecting nanometric displacements with optical ruler metrology. Science 364, 771-775 (2019).

22. Soskin, M. S. \& Vasnetsov, M. V. Singular optics. Prog. Opt. 42, 219-276 (2001).

23. Berry, M. V. \& Dennis, M. R. Knotted and linked phase singularities in monochromatic waves. Proc. R. Soc. Lond. A. 457, 2251-2263 (2001).

24. Leach, J., Dennis, M. R., Courtal, J. \& Padgett, M. J. Laser beams: knotted threads of darkness. Nature 432, 165 (2004).

25. Dennis, M. R., Holleran, K. O. \& Padgett, M. J. Singular optics: optical vortices and polarization singularities. Prog. Opt. 53, 293-363 (2009).

26. Wang, Z. B., Luk'yanchuk, B. S., Hong, M. H., Lin, Y. \& Chong, T. C. Energy flow around a small particle investigated by classical Mie theory. Phys. Rev. B 70, 035418 (2004).

27. Bashevoy, M. V., Fedotov, V. A. \& Zheludev, N. I. Optical whirlpool on an absorbing metallic nanoparticle. Opt. Express 13, $8372-8379$ (2005).

28. Luk'yanchuk, B. S., Miroshnichenko, A. E. \& Kivshar, Y. S. Fano resonances and topological optics: an interplay of far- and near-field interference phenomena. Journal of Optics 15, 073001 (2013).

29. Kuznetsov, A. I., Miroshnichenko, A. E., Brongersma, M. L., Kivshar, Y. S. \& Luk'yanchuk, B. Optically resonant dielectric nanostructures. Science 354, aag2472 (2016).

30. Wang, Z. B. et al. Optical virtual imaging at $50 \mathrm{~nm}$ lateral resolution with a white light nanoscope. Nature Communications 2, 218 (2011).

31. Luk'yanchuk, B., Paniagua-Domınguez, R., Minin, I., Minin, O. \& Wang, Z. B. Refractive index less than two: Photonic nanojets yesterday, today and tomorrow. Optical Materials Express 7, 1820-1847 (2017).

32. Permyakov, R. et al. Magnetic and Electric Hotspots with Silicon Nanodimers. Nano Letters 15, 2137-2142 (2015).

33. Gattass, R. R. \& Mazur, E. Femtosecond laser micromachining in transparent materials. Nature Photonics 2, 219-225 (2008).

34. Wu, S., Wang, G., Zhao, Y. \& Su, H. BaO-TiO 2 microwave ceramics. J. Eur. Ceram. Soc 23, 2565-2568 (2003).

35. Han, K. J. et al. Magnetization-induced optical nonlinearity in ferromagnetic GaMnAs. J. Appl. Phys. 101, 063519 (2007).

36. Li, H., Zhang, X., Liu, X., Dobrowolska, M. \& Furdyna, J. Ultrafast Modulation of Magnetization Dynamics in Ferromagnetic (Ga, Mn) As Thin Films. Applied Sciences 8, 1880 (2018).

37. Haynes, C. L., McFarland, A. D. \& Van Duyne, R. P. Surface-enhanced Raman spectroscopy. Analytical Chemistry 77, 338-346 A (2005).

38. Oraevsky, A. N. Whispering-gallery waves. Quantum Electron. 32, 377 (2002).

39. Savchenkov, A. A., Matsko, A. B., Ilchenko, V. S., Yu, N. \& Malaki, L. Microwave Photonics Applications of Whispering Gallery Mode resonators. IEEE MSMW'07 Symposium Proc., Khark 1, 79-84 (2007).

\section{Acknowledgements}

The work was supported by Center for Photonics Expertise (CPE), the European Regional Development Fund (ERDF) and the Welsh European Funding Office (WEFO), H2020 research and innovation program (grant No 737164) and partially was carried out within the framework of the Tomsk Polytechnic University Competitiveness Enhancement Program, Russia. B.L acknowledges support by the Russian Ministry of Education and Science (\#14.W03.31.0008). The authors acknowledge fruitful discussions with Ramon Paniagua-Dominguez and his proof-reading the manuscript.

\section{Author contributions}

Z.W. and B.L. led the work and developed the main ideas, programs and calculations. L.Y., B.Y., J.M., R.D., O.M., I.M., S.H., A.F. contributed to some parts of the calculations. All authors contributed to the scientific discussion and revision of the article.

\section{Competing interests}

The authors declare no competing interests.

\section{Additional information}

Correspondence and requests for materials should be addressed to Z.W. or B.L.

Reprints and permissions information is available at www.nature.com/reprints.

Publisher's note Springer Nature remains neutral with regard to jurisdictional claims in published maps and institutional affiliations.

Open Access This article is licensed under a Creative Commons Attribution 4.0 International License, which permits use, sharing, adaptation, distribution and reproduction in any medium or format, as long as you give appropriate credit to the original author(s) and the source, provide a link to the Creative Commons license, and indicate if changes were made. The images or other third party material in this article are included in the article's Creative Commons license, unless indicated otherwise in a credit line to the material. If material is not included in the article's Creative Commons license and your intended use is not permitted by statutory regulation or exceeds the permitted use, you will need to obtain permission directly from the copyright holder. To view a copy of this license, visit http://creativecommons.org/licenses/by/4.0/.

(C) The Author(s) 2019 